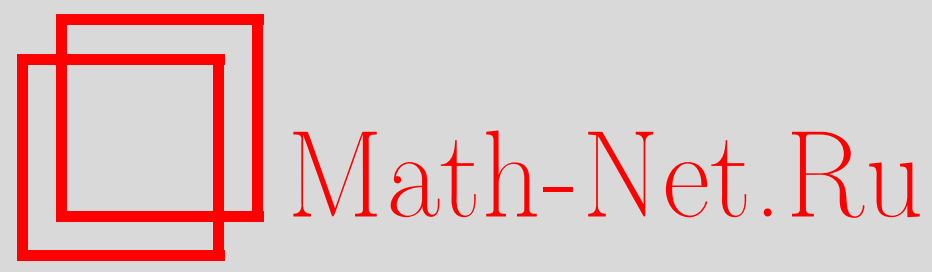

Т. Г. Хессами Пилеруд, Х. Хессами Пилеруд, Оценки снизу линейных форм от значений полилогарифмов, Матем. заметки, 2005, том 77, выпуск 4, 623-629

DOI: https://doi.org/10.4213/mzm2511

Использование Общероссийского математического портала Math-Net.Ru подразумевает, что вы прочитали и согласны с пользовательским соглашением http://www.mathnet.ru/rus/agreement

Параметры загрузки:

IP : 44.207 .124 .84

26 апреля 2023 г., 09:59:16

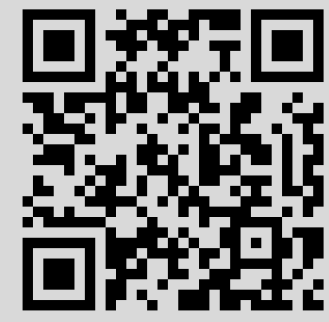


УДК 511.36

\section{ОЦЕНКИ СНИЗУ ЛИНЕЙНЫХ ФОРМ ОТ ЗНАЧЕНИЙ ПОЛИЛОГАРИФМОВ}

\section{Т. Хессами Пилеруд, Х. Хессами Пилеруд}

В работе с помощью аппроксимаций Эрмита-Паде второго рода получена оценка снизу для модуля линейной формы с целыми коэффициентами от значений полилогарифмических функций в рациональной точке. Эта оценка учитывает рост всех коэффициентов линейной формы.

Библиография: 15 названий.

Рассмотрим полилогарифмические функции

$$
L_{k}(z)=\sum_{\nu=1}^{\infty} \frac{z^{\nu}}{\nu^{k}}, \quad k \geqslant 1, \quad|z|<1
$$

В данной работе с помощю приближений Эрмита-Паде второго рода получена оценка снизу для модуля линейной формы с целыми коэффициентами от значений функций (1) в рациональной точке, зависящая от всех коэффициентов формы.

Teорема 1. Пусть $b \in \mathbb{Z}, a \in \mathbb{N}, a^{m+1} e^{m^{2}+m \ln m}<|b|$. Тогда cyществует noстоянная $c=c(a, b, m)>0$ такая, что для любых иельх чисел $x_{0}, x_{1}, \ldots, x_{m}, \boldsymbol{в}$ совокупности отличных от нуля, $u \bar{x}_{k} \geqslant \max \left(1,\left|x_{k}\right|\right), k=1, \ldots, m$, удовлетворяющих условию $\bar{x}_{1} \geqslant \bar{x}_{2} \geqslant \cdots \geqslant \bar{x}_{m}$, справедливо неравенство

$$
\begin{gathered}
\left|x_{0}+\sum_{k=1}^{m} x_{k} L_{k}\left(\frac{a}{b}\right)\right|>c \cdot\left(\bar{x}_{1} \cdots \bar{x}_{m}\right)^{-1} \cdot \bar{x}_{1}^{-\delta}, \\
\delta=\frac{m^{3}+m^{2} \ln m+m^{2}+m(m+2) \ln 2+m(m+1) \ln a}{\ln |b|-(m+1) \ln a-m^{2}-m \ln m} .
\end{gathered}
$$

СлЕДСТВиЕ 1. Пусть $b \in \mathbb{Z}, a \in \mathbb{N}, \varepsilon>0$,

$$
|b|^{\varepsilon /(m+\varepsilon)}>a^{m+1} e^{m^{2}+m \ln m+4 m} .
$$

Работа выполнена при частичной поддержке Института исследований по теоретической физике и математике (Иран), гранты № 83110020 (первый автор) и № 83110021 (второй автор). 
Тогда для любого ненулевого набора иельх чисел $x_{0}, x_{1}, \ldots, x_{m}$ и чисел $\bar{x}_{k} \geqslant \max (1$, $\left.\left|x_{k}\right|\right), k=1, \ldots, m$, удовлетворяюших условиям $\bar{x}_{1} \geqslant \bar{x}_{2} \geqslant \cdots \geqslant \bar{x}_{m}, \bar{x}_{1} \geqslant X_{0}=$ $X_{0}(a, b, m)>0$, справедливо неравенство

$$
\left|x_{0}+\sum_{k=1}^{m} x_{k} L_{k}\left(\frac{a}{b}\right)\right|>\left(\bar{x}_{1} \cdots \bar{x}_{m}\right)^{-1} \cdot \bar{x}_{1}^{-\varepsilon} .
$$

Принцип Дирихле показьвает, что уточнение последнего неравенства возможно лишь за счет величины $\varepsilon$.

Подробную историю постановки задачи можно найти в $[1$, гл. $5, \S 6]$. Отметим также, что первая оценка линейной формы, зависящая от всех коэффициентов, для чисел $e^{\alpha_{i}}$, $\alpha_{i} \in \mathbb{Q}, i=1, \ldots, m$, была получена А. Бейкером в [2]. Оценки линейных форм, зависящие от всех коэффициентов, для значений некоторых конкретных гипергеометрических $E$-функций были получены в работах [3]-[5], а для произвольного набора $E$-функций, составляюших решение системы линейных дифференциальных уравнений первого порядка и удовлетворяющих некоторьп дополнительным ограничениям, - в работе [6].

Для значений $G$-функций оценки подобного типа были доказаны лишш для некоторых совокупностей функций [7]-[11]. Теорема 1 уточняет результат работы [10]. Этого удается достичь за счет применения конструкции приближений Эрмита-Паде второго рода к полилогарифмическим функциям, а не первого как в работе [10]. Заметим, что диагональные аппроксимации Эрмита-Паде второго рода для полилогарифмов применялись в работах [12]-[14] для получения результатов об иррациональности значений функций (1) и оценок снизу соответствующих линейных форм, зависящих от максимума модуля коэффициентов формы. Функциональные свойства матричных аппроксимаций Эрмита-Паде полилогарифмов изучались в [15].

Перейдем к доказательству теоремы.

1. Построение приближающих форм. Пусть $n_{1}, \ldots, n_{m}$ - натуральные числа, $n_{1} \geqslant \cdots \geqslant n_{m}, N=\sum_{k=1}^{m} n_{k}, \mathbf{n}=\left(n_{1}, \ldots, n_{m}\right)$.

Положим

$$
R_{\mathbf{n}}(\nu)=\frac{(-1)^{N} \cdot N !}{n_{1} ! \cdots n_{m} !} \cdot \frac{\left(\nu-n_{1}\right)_{n_{1}}\left(\nu-n_{2}\right)_{n_{2}} \cdots\left(\nu-n_{m}\right)_{n_{m}}}{\nu(\nu+1) \cdots(\nu+N)}
$$

где $(\nu)_{0}=1$ и $(\nu)_{k}=\nu(\nu+1) \cdots(\nu+k-1)$ при $k \geqslant 1$.

Для $\mu=1,2, \ldots, m$ рассмотрим функции

$$
E_{\mathbf{n}, \mu}(z)=\frac{(-1)^{\mu-1}}{(\mu-1) !} \sum_{\nu=1}^{\infty} R_{\mathbf{n}}^{(\mu-1)}(\nu) z^{-\nu}
$$

Так как $R_{\mathbf{n}}(\nu)=O(1 / \nu)$ при $\nu \rightarrow \infty$, ряды в (4) абсолютно сходятся при $z \in \mathbb{C},|z|>1$. Пусть $D_{N}=\operatorname{HOK}(1,2, \ldots, N)$.

ЛЕмма 1. Для $\mathbf{n} \in \mathbb{N}^{m}, \mu=1,2, \ldots, m u|z|>1$ справедливо равенство

$$
E_{\mathbf{n}, \mu}(z)=Q_{\mathbf{n}}(z) \cdot L_{\mu}\left(\frac{1}{z}\right)-P_{\mathbf{n}, \mu}(z),
$$

əде

$$
Q_{\mathbf{n}}(z) \in \mathbb{Z}[z], \quad D_{N}^{\mu} P_{\mathbf{n}, \mu}(z) \in \mathbb{Z}[z], \quad \operatorname{deg} Q_{\mathbf{n}}(z)=N, \quad \operatorname{deg} P_{\mathbf{n}, \mu}(z) \leqslant N-1
$$

$u$

$$
\operatorname{ord}_{z=\infty} E_{\mathbf{n}, \mu}(z) \geqslant n_{\mu}+1
$$


ДокАЗАТЕльСтво. Раскладывая $R_{\mathbf{n}}(\nu)$ в сумму простейших дробей, получим

$$
R_{\mathbf{n}}(\nu)=\sum_{k=0}^{N} \frac{\omega_{\mathbf{n}, k}}{\nu+k}
$$

где

$$
\omega_{\mathbf{n}, k}=\left.R_{\mathbf{n}}(\nu)(\nu+k)\right|_{\nu=-k}=(-1)^{k}\left(\begin{array}{c}
N \\
k
\end{array}\right) \prod_{j=1}^{m}\left(\begin{array}{c}
k+n_{j} \\
n_{j}
\end{array}\right) \in \mathbb{Z} .
$$

Тогда согласно (4) имеем

$$
\begin{aligned}
E_{\mathbf{n}, \mu}(z) & =\sum_{k=0}^{N} \sum_{\nu=1}^{\infty} \frac{\omega_{\mathbf{n}, k}}{(\nu+k)^{\mu}} z^{-\nu}=\sum_{k=0}^{N} \omega_{\mathbf{n}, k} z^{k} \cdot \sum_{\nu=1}^{\infty} \frac{z^{-(\nu+k)}}{(\nu+k)^{\mu}} \\
& =Q_{\mathbf{n}}(z) \cdot L_{\mu}\left(\frac{1}{z}\right)-P_{\mathbf{n}, \mu}(z)
\end{aligned}
$$

где

$$
\begin{gathered}
Q_{\mathbf{n}}(z)=\sum_{k=0}^{N} \omega_{\mathbf{n}, k} z^{k} \in \mathbb{Z}[z], \quad P_{\mathbf{n}, \mu}(z)=\sum_{k=0}^{N} \sum_{l=1}^{k} \frac{\omega_{\mathbf{n}, k}}{l^{\mu}} z^{k-l} \in \mathbb{Q}[z], \\
\operatorname{deg} Q_{\mathbf{n}}(z)=N, \quad \operatorname{deg} P_{\mathbf{n}, \mu}(z) \leqslant N-1 .
\end{gathered}
$$

Ввиду справедливости включений (7) и

$$
D_{N}^{\mu} \cdot \sum_{l=1}^{k} \frac{1}{l^{\mu}} \in \mathbb{Z}, \quad k=1,2, \ldots, N,
$$

заключаем, что $D_{N}^{\mu} \cdot P_{\mathbf{n}, \mu} \in \mathbb{Z}[z]$.

Неравенство (6) следует из (4) и (3), и лемма доказана.

ЛЕмма 2. При $|z|>1$ справедливо неравенство

$$
\left|Q_{\mathbf{n}}(z)\right|<(N+1) \cdot 2^{(m+2) N}|z|^{N} .
$$

ДокаЗАТЕЛЬСТво. Так как согласно (7) и (8)

$$
Q_{\mathbf{n}}(z)=\sum_{k=0}^{N}(-1)^{k}\left(\begin{array}{c}
N \\
k
\end{array}\right)\left(\begin{array}{c}
k+n_{1} \\
n_{1}
\end{array}\right) \cdots\left(\begin{array}{c}
k+n_{m} \\
n_{m}
\end{array}\right) z^{k}
$$

требуемая оценка следует из неравенства $\left(\begin{array}{l}n \\ k\end{array}\right) \leqslant 2^{n}$ для всех $k=0,1, \ldots, n, n \in \mathbb{N}$.

Лемма 3. При $|z|>1$ и всех $\mu=1,2, \ldots, m$ имеет место неравенство

$$
\left|E_{\mathbf{n}, \mu}(z)\right| \leqslant \frac{2^{\mu} \cdot m^{N} \cdot|z|^{-n_{\mu}}}{|z|-1} .
$$


ДокАЗАТЕЛЬСТво. Из (4) и (6) получаем, что

$$
\left|E_{\mathbf{n}, \mu}(z)\right| \leqslant \frac{1}{(\mu-1) !} \sum_{\nu=n_{\mu}+1}^{\infty}\left|R_{\mathbf{n}}^{(\mu-1)}(\nu)\right| \cdot|z|^{-\nu},
$$

где

$$
R_{\mathbf{n}}^{(\mu-1)}(\nu)=\frac{(\mu-1) !}{2 \pi i} \oint_{|\xi-\nu|=1 / 2} \frac{R_{\mathbf{n}}(\xi)}{(\xi-\nu)^{\mu}} d \xi .
$$

Для всех целых $k$ и комплексных $\xi=\nu+e^{i \varphi} / 2,0 \leqslant \varphi \leqslant 2 \pi, \nu \in \mathbb{N}$, находим $|\xi+k|^{2}=$ $(\nu+k)^{2}+(\nu+k) \cos \varphi+1 / 4$, откуда следует, что

$$
|\nu+k|-\frac{1}{2} \leqslant|\xi+k| \leqslant|\nu+k|+\frac{1}{2} .
$$

Тогда для $\nu>1$ и $n \in \mathbb{N}$ имеем

$$
\left|(\xi-n)_{n}\right| \leqslant|\xi-1| \cdot(|\xi-1|+1) \cdots(|\xi-1|+n-1) \leqslant\left(\nu-\frac{1}{2}\right)_{n} .
$$

Далее, учитывая (3), (12) и (13), получим, что на контуре интегрирования

$$
\left|R_{\mathbf{n}}(\xi)\right| \leqslant \frac{N !}{n_{1} ! \cdots n_{m} !} \cdot \frac{(\nu-1 / 2)_{n_{1}}(\nu-1 / 2)_{n_{2}} \cdots(\nu-1 / 2)_{n_{m}}}{(\nu-1 / 2)(\nu+1 / 2) \cdots(\nu+N-1 / 2)} .
$$

Представим произведение, стоящее в знаменателе последней дроби, в следующем виде:

$$
\left(\nu-\frac{1}{2}\right)\left(\nu+\frac{1}{2}\right) \cdots\left(\nu+N-\frac{3}{2}\right)=\left(\nu-\frac{1}{2}\right)_{n_{1}} \cdots\left(\nu-\frac{1}{2}+n_{1}+\cdots+n_{m-1}\right)_{n_{m}} .
$$

Тогда

$$
\left|R_{\mathbf{n}}(\xi)\right| \leqslant \frac{N !}{n_{1} ! \cdots n_{m} !} \cdot \frac{1}{\nu+N-1 / 2} \leqslant \frac{m^{N}}{\nu+N-1 / 2} .
$$

Учитьвая (11), окончательно получаем

$$
\left|R_{\mathbf{n}}^{(\mu-1)}(\nu)\right| \leqslant \frac{(\mu-1) ! \cdot 2^{\mu-1} \cdot m^{N}}{\nu+N-1 / 2} \leqslant \frac{(\mu-1) ! \cdot 2^{\mu} \cdot m^{N}}{\nu+N},
$$

и утверждение леммы теперь следует из (10).

Определим набор мультииндексов

$$
\mathbf{n}_{j}=\left(n_{1}+1, \ldots, n_{j}+1, n_{j+1}, \ldots, n_{m}\right), \quad j=0,1, \ldots, m .
$$

Рассмотрим определитель

$$
\Delta_{\mathbf{n}}(z)=\left|\begin{array}{cccc}
Q_{\mathbf{n}_{0}}(z) & P_{\mathbf{n}_{0}, 1}(z) & \ldots & P_{\mathbf{n}_{0}, m}(z) \\
Q_{\mathbf{n}_{1}}(z) & P_{\mathbf{n}_{1}, 1}(z) & \ldots & P_{\mathbf{n}_{1}, m}(z) \\
\vdots & \vdots & \ddots & \vdots \\
Q_{\mathbf{n}_{m}}(z) & P_{\mathbf{n}_{m}, 1}(z) & \ldots & P_{\mathbf{n}_{m}, m}(z)
\end{array}\right|
$$


ЛЕмма 4. Выполнено

$$
\Delta_{\mathbf{n}}(z) \equiv \text { const } \neq 0
$$

ДокАЗАТЕЛЬСТво. Будем нумеровать строки и столбцы определителя (14) индексами от 0 до $m$. Прежде всего заметим, что этот определитель - многочлен. Далее, для каждого $j=1, \ldots, m$ вычтем из $j$-го столбца определителя $\Delta_{\mathbf{n}}(z)$ нулевой столбец, умноженный на $L_{j}(1 / z)$. Согласно (5) получим

$$
\Delta_{\mathbf{n}}(z)=(-1)^{m}\left|\begin{array}{cccc}
Q_{\mathbf{n}_{0}}(z) & E_{\mathbf{n}_{0}, 1}(z) & \ldots & E_{\mathbf{n}_{0}, m}(z) \\
Q_{\mathbf{n}_{1}}(z) & E_{\mathbf{n}_{1}, 1}(z) & \ldots & E_{\mathbf{n}_{1}, m}(z) \\
\vdots & \vdots & \ddots & \vdots \\
Q_{\mathbf{n}_{m}}(z) & E_{\mathbf{n}_{m}, 1}(z) & \ldots & E_{\mathbf{n}_{m}, m}(z)
\end{array}\right| .
$$

Пусть $\Delta_{\mathbf{n}, j}(z), 0 \leqslant j \leqslant m,-$ алгебраическое дополнение элемента $Q_{\mathbf{n}_{j}}(z)$ определителя (15):

Тогда

$$
\Delta_{\mathbf{n}, j}(z)=(-1)^{j}\left|\begin{array}{ccc}
E_{\mathbf{n}_{0}, 1}(z) & \ldots & E_{\mathbf{n}_{0}, m}(z) \\
\ldots \ldots \ldots \ldots \ldots \ldots & \ldots \ldots \ldots \ldots \\
E_{\mathbf{n}_{j-1}, 1}(z) & \ldots & E_{\mathbf{n}_{j-1}, m}(z) \\
E_{\mathbf{n}_{j+1}, 1}(z) & \ldots & E_{\mathbf{n}_{j+1}, m}(z) \\
\ldots \ldots \ldots \ldots \ldots \ldots \ldots \ldots \ldots \ldots \\
E_{\mathbf{n}_{m}, 1}(z) & \ldots & E_{\mathbf{n}_{m}, m}(z)
\end{array}\right| .
$$

$$
\Delta_{\mathbf{n}}(z)=(-1)^{m} \sum_{j=0}^{m} Q_{\mathbf{n}_{j}}(z) \Delta_{\mathbf{n}, j}(z) .
$$

Согласно лемме 1 находим

$$
\begin{gathered}
\operatorname{deg} Q_{\mathbf{n}_{j}}(z)=N+j, \quad j=0,1, \ldots, m, \quad \Delta_{\mathbf{n}, 0}(z)=O\left(\frac{1}{z^{N+m+1}}\right), \quad z \rightarrow \infty, \\
\Delta_{\mathbf{n}, j}(z)=O\left(\frac{1}{z^{N+m}}\right), \quad z \rightarrow \infty, \quad j=1, \ldots, m .
\end{gathered}
$$

Из (16)-(18) вытекает, что $\Delta_{\mathbf{n}}(z)=O(1)$ при $z \rightarrow \infty$, т.е. $\Delta_{\mathbf{n}}(z)-$ многочлен нулевой степени: $\Delta_{\mathbf{n}}(z) \equiv \Delta_{\mathbf{n}}(0)$. Учитывая $(16),(9),(4),(3)$, окончательно получаем

$$
\Delta_{\mathbf{n}}(z) \equiv \Delta_{\mathbf{n}}(0)= \pm \prod_{k=1}^{m}\left(\begin{array}{c}
N+m+n_{k}+1 \\
n_{k}+1
\end{array}\right) \cdot \prod_{j=0}^{m-1} \frac{1}{j !} R_{\mathbf{n}_{j}}^{(j)}\left(n_{j+1}+1\right) \neq 0
$$

и лемма доказана.

2. Доказательство теоремы 1. Пусть $z=b / a$, где числа $a \in \mathbb{N}$ и $b \in \mathbb{Z}$ удовлетворяют условию

$$
|b|>a^{m+1} \cdot e^{m^{2}+m \ln m} .
$$

Тогда, в частности, $|z|>1$. Рассмотрим форму

$$
\ell=x_{0}+x_{1} L_{1}\left(\frac{a}{b}\right)+\cdots+x_{m} L_{m}\left(\frac{a}{b}\right)
$$


с произвольными цельми коэффициентами, невсе из которых равны нулю, и пусть числа $\bar{x}_{k} \geqslant \max \left(1,\left|x_{k}\right|\right), k=1, \ldots, m$, таковы, что $\bar{x}_{1} \geqslant \cdots \geqslant \bar{x}_{m}$. Определим натуральное число $n_{1}$ как наименьшее, удовлетворяющее условию

$$
\bar{x}_{1} \cdot\left(e^{m^{2}+m \ln m} \cdot \frac{a^{m+1}}{|b|}\right)^{n_{1}}<\frac{1}{2 m} .
$$

В силу (19) при достаточно большом $\bar{x}_{1}$ такое $n_{1}$ всегда существует. Для $1<k \leqslant m$ определим $n_{k}$ как наименьшее натуральное, удовлетворяющее условию

$$
\bar{x}_{k} \cdot\left(e^{m^{2}+m \ln m} \cdot a^{m}\right)^{n_{1}} \cdot\left(\frac{a}{|b|}\right)^{n_{k}}<\frac{1}{2 m} .
$$

Легко видеть, что при таком выборе чисел $n_{k}$ вьполнено $n_{1} \geqslant n_{2} \geqslant \cdots \geqslant n_{m}$. Кроме того, при фиксированных $a$ и $b$ условия $\bar{x}_{1} \rightarrow \infty, n_{1} \rightarrow \infty$ и $N \rightarrow \infty$ эквивалентны. Далее по лемме 4 найдется такое $j=0,1, \ldots, m$, зависящее от $\mathbf{n}$, что

$$
x_{0} Q_{\mathbf{n}_{j}}\left(\frac{b}{a}\right)+\sum_{k=1}^{m} x_{k} P_{\mathbf{n}_{j}, k}\left(\frac{b}{a}\right) \neq 0 .
$$

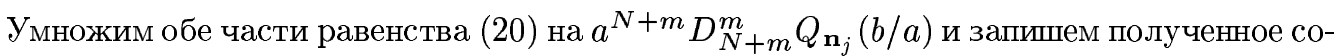
отношение в виде

$$
\begin{aligned}
a^{N+m} D_{N+m}^{m} Q_{\mathbf{n}_{j}}\left(\frac{b}{a}\right) \ell= & a^{N+m} D_{N+m}^{m}\left(x_{0} Q_{\mathbf{n}_{j}}\left(\frac{b}{a}\right)+\sum_{k=1}^{m} x_{k} P_{\mathbf{n}_{j}, k}\left(\frac{b}{a}\right)\right) \\
& +a^{N+m} D_{N+m}^{m} \sum_{k=1}^{m} x_{k} E_{\mathbf{n}_{j}, k}\left(\frac{b}{a}\right) .
\end{aligned}
$$

В силу леммы 1 первое слагаемое в правой части (22) есть целое число, а в силу (21) это число отлично от нуля. Следовательно, абсолютная величина этого числа не меньше единицы. Тем самым, мы можем написать неравенство

$$
a^{N+m} D_{N+m}^{m}\left|Q_{\mathbf{n}_{j}}\left(\frac{b}{a}\right)\right| \cdot|\ell| \geqslant 1-a^{N+m} D_{N+m}^{m}\left|\sum_{k=1}^{m} x_{k} E_{\mathbf{n}_{j}, k}\left(\frac{b}{a}\right)\right| .
$$

Согласно леммам 2,3 при $|z|>1$ и всех достаточно больших $N$ справедливы оценки

$$
\begin{gathered}
a^{N+m} D_{N+m}^{m} \leqslant A \cdot e^{m N} a^{N} \\
\left|Q_{\mathbf{n}_{j}}\left(\frac{b}{a}\right)\right| \leqslant B \cdot 2^{(m+2) N}\left(\frac{|b|}{a}\right)^{N}, \quad\left|E_{\mathbf{n}_{j}, k}\left(\frac{b}{a}\right)\right| \leqslant C \cdot m^{N}\left(\frac{|b|}{a}\right)^{-n_{k}},
\end{gathered}
$$

где $A=e^{o(N)}, B=e^{o(N)}, C=e^{o(N)}$ при $N \rightarrow \infty$.

Учитывая неравенства (23), для всех достаточно больших $N$ находим

$$
e^{m N} a^{N} \cdot 2^{(m+2) N}\left(\frac{|b|}{a}\right)^{N}|\ell| \geqslant 1-e^{m N} a^{N} \sum_{k=1}^{m} \bar{x}_{k} m^{N}\left(\frac{a}{|b|}\right)^{n_{k}} .
$$


Учитьвая выбор чисел $\bar{x}_{k}, k=1, \ldots, m$, получаем, что для всех $n_{1}$, больших некоторого числа, зависящего от $a$ и $b$, правая часть неравенства (24) будет больше $1 / 2$, т.е.

$$
\begin{aligned}
|\ell| & >\frac{1}{2} e^{-(m+(m+2) \ln 2) N}|b|^{-N} \\
& >c\left(\bar{x}_{1} \cdots \bar{x}_{m}\right)^{-1} e^{-\left(m^{3}+m^{2} \ln m\right) n_{1}} a^{-N-m^{2} n_{1}} e^{-(m+(m+2) \ln 2) N} \\
& >c_{*}\left(\bar{x}_{1} \cdots \bar{x}_{m}\right)^{-1}\left(a^{m^{2}+m} e^{m^{3}+m^{2} \ln m+m^{2}+m(m+2) \ln 2}\right)^{-n_{1}} \\
& >c_{* *}\left(\bar{x}_{1} \cdots \bar{x}_{m}\right)^{-1} \cdot \bar{x}_{1}^{-\delta}
\end{aligned}
$$

где $c_{* *}=c_{* *}(a, b, m)$ и $\delta$ определено в (2). Теорема доказана.

\section{СПИСОК ЦИТИРОВАННОЙ ЛИТЕРАТУРЫ}

[1] Fel'dman N. I., Nesterenko Yu. V. Transcendental Numbers. Number Theory IV. Encyclopaedia of Math. Sci. V. 44. Berlin: Springer-Verlag, 1998.

[2] Baker A. On some Diophantine inequalities involving the exponential function // Canad. J. Math. 1965. V. 17. P. 616-626.

[3] Фельдман Н. И. Оценки снизу для некоторых линейных форм // Вестн. МГУ. Сер. 1. Матем., мех. 1967. № 2. С. 63-72.

[4] Väänänen K. On lower estimates for linear forms involving certain transcendental numbers // Bull. Austral. Math. Soc. 1976. V. 14. P. 161-179.

[5] Väänänen K. On linear forms of the values of one class of $E$-functions // Acta Univ. Ouluen. Ser. A. 1976. № 41. P. 1-19.

[6] Зудилин В.В.Об оценках снизу многочленов от значений некоторых целых функций // Матем. сб. 1996. Т. 187. № 12. С. 57-86.

[7] Фельдман Н. И. Об одной линейной форме // Acta Arith. 1972. V. 21. P. 347-355.

[8] Сорокин В.Н. Об иррациональности значений гипергеометрических функций // Матем. сб. 1985. Т. $127(169)$. № 2(6). С. 245-258.

[9] Галочкин А. И. Оценки снизу линейных форм от значений некоторых $G$-функций // Матем. заметки. 1975. Т. 18. № 4. С. 541-552.

[10] Хессами Пилеруд Т. Оценка снизу одной линейной формы // Матем. заметки. 1999. Т. 66. № 4. С. $617-623$.

[11] Хессами Пилеруд Т. Оценки снизу линейных форм от значений некоторых гипергеометрических функций // Матем. заметки. 2000. Т. 67. № 3. С. 441-451.

[12] Chudnovsky G. V. Padé approximations to the generalized hypergeometric functions. I // J. Math. Pures Appl. 1979. V. 58. P. 445-476.

[13] Гутник Л.А. О линейной независимости над $\mathbb{Q}$ дилогарифмов в рациональных точках // УМH. 1982. Т. 37. № 5. С. 179-180.

[14] Hata M. On the linear independence of the values of polylogarithmic functions // J. Math. Pures Appl. 1990. V. 69. № 2. P. 133-173.

[15] Сорокин В. Н. Аппроксимации Эрмита-Паде полилогарифомов // Изв. вузов. Матем. 1994. № 5. C. 49-59.

Shahrekord University, Shahrekord, Iran

Поступило

E-mail: hessamit@ipm.ir

28.01.2004

Исправленный вариант

29.06 .2004 